\title{
Can Field Theory be Applied to the Semiotics of Communication?
}

The title of this study is adapted from the title of the dissertation that Robert S. Hartman submitted at the end of 1946 to the Graduate School of Northwestern University "in partial fulfillment of the requirements for the degree Doctor of Philosophy" (Can Field Theory be Applied to Ethics?). Although the path I shall follow is quite different, references to Hartman's unpublished thesis will appear. This shows that trying to answer such a question means to elaborate on the epistemological level of the subject matter, in this case communication. The attempt presented below should not be understood as yet another in the series of the so-called scientization of the humanities, since communication theories never really lacked a scientific foundation of one sort (information theory) or another (linguistic, structuralistic, logical, etc.). Actually, the question posed in the title involves the obligation of setting communication into its proper framework. Field, as we shall see, means interaction, or action at distance, geometry, or frame of reference. The latter meaning is of particular importance in this preliminary stage, communication having its proper frame of reference within semiotics. This is a strong affirmation, one which might better have been postponed until a certain methodology was set up. I choose to introduce it in the preliminaries because it is commonplace in today's semiotics, and I shall come back to it at least because I know that sometimes such commonplace notions turn out to be false friends. Originally, Field was applied in describing concretely observable bi-polar physical phenomena. Dewey suggested the comparison of situations to physical fields, considering that scientific inquiry is problems-solving in situations, and introduced the dual model of the field. "Situations of inquiry are states of energy, tension existing between two poles," Hartman "later affirms (1946 p.4), a limitation which this study will not accept for reasons clear enough to those familiar with Peirce's sign definition. (I will return to this, too.)

The framework of semiotics, i.e., the framework in which sign processes are to be discerned, is what we traditionally call culture. Situating sign processes into the frame in which their meaning is made evident means to relate them to the conceptual aspect of the field on the one hand, and to its practical aspect on the other. Consequently, the field has an ideal and a real pole; the need for their assembling in a consistent structure represents the third term. Peirce, whose semiotics is built on the foundation of his philosophy, noticed that the correct 
progression is Possible-Real-Necessary. Accordingly, one can paraphrase Hartman (1946, p. 22) an ascertain: When the setting up of an idea as a potential framework for a phenomenon and the application of that framework in action prove in practice to be necessary, we have the ideal-real-necessary field of semiotics. Not any setting of sign processes into framework is necessary; the degree of necessity can be proven either historically or methodologically. Because we cannot check our semiotic concepts and the results in a time capsule in which every other type of influence (in the generalized social field) can be modelled, it remains that methodology, i.e., the logical status, must be improved. It becomes only natural to verify the consistence, or the indepence, decidability, categoricity, duality, etc. of a semiotic theory. This is an important goal to be reached in future research and studies. Here we limit ourselves to a preliminary problem and shall try to define the semiotic field and then, if the attempt proves successful, the complementary fields of communication and signification.

Specialized literature often brings up the concept of the semiotic field without determining this field, however, without even attempting a justification (epistemological or otherwise) for using the term, which comes from physics. The field theory points out the importance of the fact that each event taking place in the field is a result of a number of factors, but the theory itself is not reduced to pointing out these multideterminations. Furthermore, recognition of these multideterminations as well as their representation in a phase space model (a space with a number of dimensions equal to the factors influencing an event) does not necessarily presuppose a field theory. It should be understood as a method for analyzing causal relations and for realizing "scientific constructions" (Lewin, 1939 , p. 307), expressed mathematically in the sometimes difficult terms of differential geometry (Spivak, 1970), which semiotics cannot take upon itself. The proposed aim basically imposes and represents a specific definition, the elaboration of concepts (used much to freely elsewhere), and a suggestion of a formalism adapted to the object. In this sense, some basic elements will be summarized below as a methodological premise needed to fulfill the above mentioned aim.

In his discussion of "The Nature of Some of our Physical Concepts," Bridgman (1952) characterizes the field as "one of the foundation blocks of modern physics," outlining its history in one sentence: "invented by Faraday's intuitional genius, mathematically robed by Maxwell, and crowned by Einstein in his theory of relativity." The field "absolves us from accepting that intellectual monstrosity of long-distance action." At the extreme, these elements are sufficient not only for understanding the context field theory appeared in but also for explaining its extension from physics to the humanities. Newton expressed in a relatively rhetorical manner the conviction that action between two bodies is transmitted trough something. Faraday drafted the power lines of the action of transmission and observed that there are qualitative differences between the electric and magnetic fields. Let us recall only well-known concepts: power lines can be shown 
in simple experiments (for example, the one in which iron filings gather around a magnet), likewise for the connection between the electric and magnetic fields. Quantitative relations express interactions in the electromagnetic field. In the case of the gravitational field, matters are the same in principle.

One of the first attempts at extending the field model to the humanities was undertaken by Kurt Lewin (1952) in psychology, an attempt of demonstrating that this was not a matter of a metaphor taken from scientific vocabulary but an effective means of research, analysis, and determinations of quantitative and qualitative aspects of psychological processes. It is likely that any current attempt to apply the concept under discussion to a domain other than psychology will have to refer to the way in which Lewin justified the psychological field in its quality as a field of behavior. Attempts have been made at applying this model to other disciplines: biology (cf. Gurwich, Spemann), sociology (cf. Lundberg, Brown) aesthetics (but inadequately so). One limitation must be imposed here: never, to our knowledge, has an analogy between the physical (gravitational, electric, or magnetic) field and the spiritual (cultural, psychological, semiotic, etc.) been made in the direction of setting up rigid parallels between electric charges, magnets, gravity, motion, or velocity, for instance, and psychic factors, moral values, signs, or other entities of this nature. Asking himself whether field theory could be applied to ethics, Hartman likewise avoided applying mechanics or forced extensions of analogies. In semiotics, its is to the merit of Eco (1968), (1976) and Lotman (1968) for having insisted on the field condition of semiotic (especially cultural) reality. In one of his early works (Opera apperta), Eco proposed the model of the ideological field as the open field of the existence and fulfillment of aesthetic work. But this was more a matter of intuitive representation, sometimes disputible, sometimes directly erroneous or gratuitous. Often in these attempts, the effective application of the field to the subject in question is missing; the intuitive level is barely transcended; the gnoseological and epistemological limits of using analogy are not specified. In addition, the field has been considered somewhat independent of the actual fact of semiotics (the sign in the dynamics of its fulfillment), which has led to an inability to distinguish between the semiotic and cultural fields, or further between the cultural and ideological fields. Thus arises the problem of bringing about a cognitive model with a precise epistemological status, starting out from a question of principle which any field theory approaches: long-distance action/interaction. But in semiotics, we must be prudent in our use of the word distance which should not be understood only in physical space as a geometric distance between two signs or groups of signs but in its generality (distance in time included). Of course, returning to Newton's exclamation, it seems evident that signs interact not in a vacuum (and here a specifically semiotic vacuum, not a physical one, of a total absence of signs) but in the space of human existence, semiotic space being one of the concrete forms of the realization of this space. If we now refer to the field model of Einstein's theory, we can extend the idea of the curving of space; we can 
even speak of movement on a geodesic. But the mechanical transfer of these terms to semiotics will not lead to real gnoseological progress.

It is known that each sign influences the sign reality it appears in and is in turn influenced by this reality. This affirmation already involves the notion that the subject is part of the sign produced and perceived. In this sense, the model of curving semiotic space is the image of this action, and the model of movement on a geodesic corresponds to the way in which sign processes take place. We know that semiotic dynamics is complicated, even more complicated in reality than the dynamics of the physical systems described by the theory of relativity. There are, however, certain cases in which the results of one scientific theory can be applied to another type of phenomena without it being affirmed that the latter is reducible in principle to physical reality but only to the possibility of analogy or modelling. Proceeding to the quantum theory of the field, we can likewise observe that analogies are possible (sometimes even necessary) between the way in which the semiotic field (as a continuum) interacts with the sign (having the character of component, part, appearance, singularity, hence discrete) and the complementary character of the field and quanta. Finally, the terminology of the field theory also seems adapted to extension; intensity, tension, flow, power line - to mention a few terms - are currently accepted almost without reserve (sometimes with too much ease).

The methodological preliminaries given up to here were dictated by necessities due primarily to the epistemological condition of the analogy and to terminological ethics. Basically, any theory containing the mathematical concept of field (scalar, verctorial, tensorial, or spinorial) and which is founded on the recognition of a principle of action/interaction can be called a field theory. We can distinguish between the material field theory (the field of the velocity of a material particle, for example), the mathematical field (desribing the velocity of disturbance, for example, making an abstraction from material particles), and mixed theory (such as the quantum theory). In order to determine the semiotic field, we must establish its character. Without restating the definition of sign, let us observe that it deals with a field in which the material bearer (the sign as sign, the representamen, the significant, etc.) can be perfectly determined statically as well as dynamically. But what defines the field is not only this bearer but the semiotic process as a material and spiritual process. Therefore, the mathematical (or pure mathematical) field only partially represents semiotic processes. The complementary, mixed field - in which the sign's structure, as it results from the categorical model, is overtaken in the contradictory whole of the component parts - gives adequate representation. Here the same philosophical problem that has often accompanied field theory reappears: is the field concept justified as long as the field is in evidence only through observations which modify it in the final analysis (object and means of observation constituting a whole)? In direct reference to semiotics, we can observe the dynamics of signs with the help of signs, hence modifying the semiotic field. Here reappears that danger of epistemological 
subjectivism that leads to the idea that the field depends on the way it is measured, determined, and observed, that it exists as the reflex of our attempts to define it. The semiotic field corresponds to the image of action, reaction, and interaction of signs, not in space per se, but in that of social existence in which man himself is part of its signs, is a sign (cf. Peirce). Its definition is not outright nor expressible in numbers which should define the semiotic state, but through its effects, through the human practice of which it is a component. This is a matter of a complex field in which there are areas that can be studied as follows:

1) linear fields-for example, the semiotic fields of institutionalized signs, such as those of traffic, in which simple principles of superimposition are applied (a sign of prohibition plus a sign or signs specifying what is prohibitted);

2) non-linear fields-for example, the reciprocal influence of different semiotic realities (verbal, nonverbal) or that within the same reality (blank verse and rhyme) as a distant influence;

3) time-dependent fields-for example, the historical evolution of some sign systems (including those of natural language);

4) time-independent fields-such as natural signs (fossils, moss on tree trunks, astronomic and meteorological signs);

5) normed fields-such as technical signs, the signs of normative functions assumed by the state, church, military, etc. Norms can be specified explicitly (as on geographical maps where a scale is given) or implicitly through the functioning of the sign system (as in church hierarchy);

6) free fields-for example, the signs of some modern art forms ("free" should not be confused with "arbitrary");

7) attributive fields-such as the signs expressing quality;

8) relational fields-such as signs that express relating (mathematical, logical, sensorial, etc.) or which take the place of a relation;

9) functional fields-for example, the sign system through whose functioning a certain function is fulfilled (signaling, knowledge, sensitivity, etc.). Any sign system fulfills a certain function, but the characteristic of functional stems from the evidence of the function fulfilled (aesthetic, logical, ethical, etc.);

10) actional fields-such as signs of praxiological systems;

11) processional fields-such as signs defining processes or representing their taking place. Not only technical processes but also social, cultural, and other should by included here;

12) discrete fields-for example, the discrete system of signs (homogenous or not) of some progressive educational forms or the system, discrete by nature, of some institutionalized signs;

13) continous fields-for example, the field of the signs of language or the sign system of time and space.

Although the main types are listed above, we are sure that the field typology is not exhausted. We also believe that no typology pertinent to signs can be constructed and verified without considering field typology. This idea is founded 
on the fact that the sign is identified in relation to its functioning and that this functioning is objectified in the reality of the field. Let us here recall "the three fields of the interpretant," as Rethore calls them in developing one of Peirce's ideas on the types of interpretants. In short, it deals with a) the immediate interpretant (the non-analyzed effect produced by signs), b) the dynamic interpretant (the sign's real effect as a unique, real event), and c) the final interpretant (the sole interpretive result to be reached if the sign is sufficiently analyzed). Furthermore, these three fields (as Rethoré calls them in misuse of the term) also show the division in two of the dynamic interpretant (as deducted or not from colateral experiments, in particular as it is derived through abduction or deduction), and in three of the final interpretant. The latter can be thematic (corresponding to the general, collective, interpretive tendency of signs as determined through abduction), dicentic (the experimentally controlled, specialized tendency of sign interpretation as determined through induction), or argumental (systematic interpretation, leading to a model; determined deductively).

We return here to the criticism made of the way in which some semioticians (not just Eco (1972), (1976), although reference will be made chiefly to his works because the terminology of the semiotic field is taken from him in particular or from Lotman along Eco's line) introduce and use the field concept. Asking whether semiotics should be considered as a specific discipline with its own unitary method and a well-defined object or as a field of research, Eco introduces confusion, which is rediscovered in many texts on semiotics. In particular, he speaks of "field" in the sense of "domain." That is, he refers to the field of semiotics for which his description in the form of a commented bibliography (taken from "La Struttura Assente" for "A Theory of Semiotic") becomes acceptable, if not totally justified. This negligence in expression would not be worth much attention if its direct consequence were not the ruining of the possibility of effectively utilizing the field model. It is clear that the disjunction field/discipline does not help in the real definition of the epistemological status of semiotics. Furthermore, the transformation of field into a system by conferring or pointing out a structure $(E c o, 1976$, p. 9$)$ is again the expression of an approximative manner of using precise concepts, hence a source of error. It is fitting to return to the way in which Lewin applied the field method to psychology, keeping in mind Hartman's specifications concerning the ethical field (a field of aspiration, of pure potentiality). Lewin expresses the so-called "Principle of Contemporaneity and the Effect of Past and Future" (1943): "Any behavior or change in a psychological field depends only on the psychological field at the moment considered." We will anticipate here that this type of dependence is expressed in the mathematical theory of Markov, a theory that offers the description of processes of communication. In the case of closed systems, there appears the possibility of referring to the past or future due precisely to the fact that nothing affects the state of the system from the outside. It has been seen, though, that the generalized semiotic system is open, a system with a history, and that it must 
consequently be discribed with the aid of an adequate mathematical apparatus. Certain subsystems of the generalized semiotic system can be dealt with on the basis of the principle of contemporaneity because they behave, in relation to the duration of direct human experience, as closed systems, (language, normed sign systems, religious sign systems, etc.). For these, semiosis-the elementary unit of the semiotic field-is the direct function of the field's state

$$
\mathrm{s}^{\mathrm{t}}=\mathrm{F}\left(\mathrm{S}^{\mathrm{t}}\right)
$$

The evidence of history or the semiotic consequences of a sign process goes back to determining functions of the type

$$
s^{t}=F\left(S^{t}\right)
$$

in particular

$$
s^{t}=F\left(S^{t+n}\right)
$$

Such determinations were frequently carried out in studies in morphology, etymology, or archaeology (to refer to the best known sign system) or in research in futurology in order to point out evolutive processes, meaningfully expressed on the semiotic level. Semiosis can also be defined through the present tense testing of semiotic situations, that is, defining the properties of a situation $S^{t}$ (for example, the semiotic repertory of institutionalized forms of power, art, or labor protection rules, or of the repertory of military signs). There are cases in which historic procedure is preferable or even the only one possible, as in the case of exhausted semiotic events like some artistic forms, languages, ideologies, etc. which have disappeared in the meantime. In other cases, present testing is necessary to avoid the uncertainty of historical conclusions. The essential fact is that the semiotic system does not reduce itself to the structure of domain (as Eco and many others believe) but represents the manner in which the parts interact, the manner in which the whole functions in relation to other systems (determinant or determined). The definition of semiotics cannot be inducted from the system structure of the domain, just as the elaboration of a semiotic model (when this appears as a discipline $(E c o, 1968))$ that would maintain which aspects do or do not belong to the discipline is illusory. The field of the interpretant, which deserves a systematic interpretation instead of the short presentation given here, still separates one aspect of the semiotic field (the dynamics of the interpretant subsign) which, although important (especially in the semiotics of interpretation), cannot take the place of the whole. The analysis of hypersigns that are realized in the semiotic field necessarily bring the interpretant back to the sign's triadic unity.

The meaning of a semiotic event can be established only on considering a certain period which depends on the nature of the situation. Thus, in the framework of semiotic subsystems of natural languages, a longer period must be kept in mind while in other subsystems in which change is rapid, a relatively short interval can be considered. The relative position of a sign or a sign complex in the semiotic field (the sign/signs of power, of new social relations, of some forms of culture, education, etc.) is established by attributing scalar values (simple numbers), that is, by establishing hierarchies (expressed through these numbers) and 
observing their modification. It is known that the sign of the swastika, for example, dominated an epoch. Other signs stemming from the organization and functioning of fascistic societies have occupied a certain position in the semiotic hierarchy of time. Signs of domination (discrimination, for instance) have existed alongside signs of dominance. Other examples can be given. In the end, the establishment of hierarchy, of modifications (the $S S$ sign in relation to the sign of the Wehrmacht) is a strictly statistical operation which becomes relevant at a certain level of considering semiotic reality, of its integration in the given reality. The relative position of words, of social formulas, of political or cultural signs used during a given period is expressed through a scalar field. The vectorial field-that of modifications, of the velocity of these modifications, of the direction these modifications take-is more important. The sign system of fashion is not just a matter of time (such as the seasons) but of the way one goes from one type of representative sign (grace, distinction, nobility, for instance) to other types (negligence, apathy, vulgarity), when one or the other appears, its relation to signs of other fields (social, political, cultural, economic, etc.) what meaning is actually brought about, and the relation between the real and apparent meaning ("democratization" or "aristocratization" of fashion).

In certain epochs, the semiotic field displays a high degree of stability, in others, a high degree of evolution and revolution. In this case, vectors (simply stated, arrows indicating values through their length and direction, the orientation of their heads) are attributed to sign complexes. The appearance and disappearance of some signs is expressed in the vectorial semiotic field. Thus is explained the vectorial representation or application of the mathematical graph theory to comparisons of variants of folkloric production. The connection between the mathematical method and the object analyzed is significant on the semiotic level. Through the tensorial and spinorial fields, we can generalize that which belongs to the multidimensionality and multidetermination of the semiotic field, but now we shall point out only the possibility of this generalization. We shall add, as a final introductive methodological fact, that we can always approach the semiotic field on the microstructural or macrostructural levels, that is, on however small differential intervals or however large integral intervals or even realize the unity of the micro- or macrostructural analysis.

The sign, as a unity around which the semiotic field is constituted, actually fulfills two functions: communication and signification. These functions-impossible to separate in the reality of the semiotic field-are displayed in the dynamics of sign processes that actually justify the sign. Therefore, the elementary unit of the field, likewise considered in its dynamics, is semiosis.

Definition 1: The behavior of a sign/set of signs in the semiotic field is called semiosis. This definition repeats affirmations made above, not only in order to avoid terminological confusion but also to point out the way we shall proceed in defining the field. In the end, the field represents a formal structure: the semiotic power lines (between signs that "attract" or "repel" or are "indifferent") are not 
physical in nature nor are they mathematical abstractions per se. From long experience, we know exactly that a struggle, for example, takes place in some cultures, that artistic, social, or ideological evolution shows moments of tension which the semiotic field faithfully reflects. These expressions of animistic nuance are used here under the premise that the sign integrates the subject, that the subject itself is a sign. Other examples can be given even for simple signs. This is not an epistemological guarantee but a confirmation of the proposed direction. According to the categorical model of the sign, approach of the semiotic field is not only motivated, but also necessary.

The distinction between communication and signification can be shown to a great extent through the model of communication as defined in the information theory (Shannon, 1959, Weaver, 1959). This model, frequently evoked and more recently criticized because its statistical aspect limits it to the syntactic level, deals with communication as a unidirectional process (from the source of information-the emitter-to a receptor). In reality, communication presupposes a twoway (dependent, independent) informational flow, and when it takes place among human beings or in their presence, it is simultaneously carried on as signification.

In order to understand the mechanism of communication and the way it is constituted with a well-determined semiotic status, let us observe that the model of the informational channel, while indicating direction by means of arrows, corresponds rather to realizations which we could here call (anticipating a more rigorous definition) the informational field generated by $\mathrm{E}$.

Figure 1

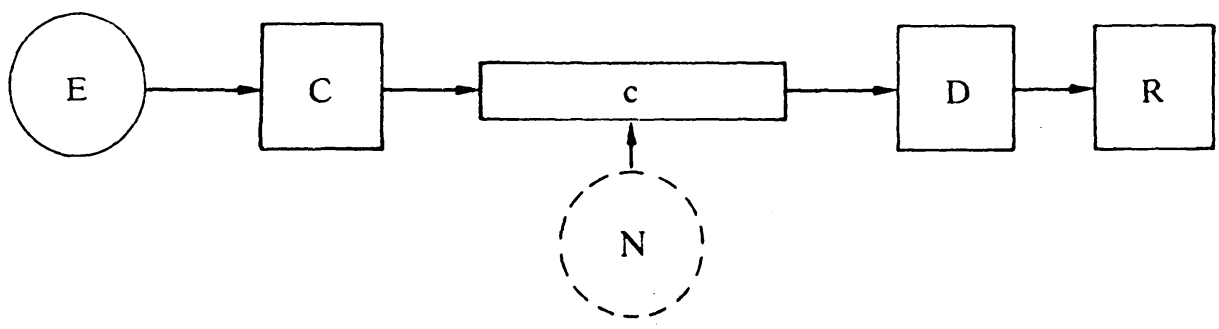

E - emitter (source of information according to Shannon)

$\mathrm{N}$ - noise (considered as generator, source)

C - codifier (transmitter, which operates on the message in some way to produce a signal suitable for transmission over the channel)

c - communication channel ("the medium used to transmit the signal")

$\mathrm{D}$ - decodifier (receiver, performs the inverse operation of that done by the transmitter)

$\mathrm{R}$ - receptor 
The analogy to a model form physics (the physics of propagation) in which the notion of field plays a fundamental role (in the classic and quantic models) has already been justified. When the analogy is extended, we shall bring further justifications. Here it should only be added that Shannon gave a mathematical description to the information source based on a statistical model (inspired by the reality of language as a sign system), defining the whole process as a Markov type of stochastic process. (The principle of contemporaneity is implicit here.) There is a finite number of possible "states," $S_{1}, S_{2}, \ldots S_{n}$. In addition, there is a set of transition probabilities $p_{i}(j)$ that defines the probability that the system, if in state $S_{1}$ go over to state $S_{j}$ (Shannon, 1959, p. 15). Without entering into detail, we should observe that the model given makes the transition for the continuum of the message to the discrete succession of signs and that it also gives a mathematical description which permits the solution of some delicate technical problems (concerning the capacity of informational systems or their optimal dimensioning, for example) as well as the approaching of the role of signs in constituting semantic units (statistical methods in linguistics, text theory, aesthetics, etc.). What interests us here is not the technical aspect of calculus nor the syntactic aspects it points out, but the way in which a semiotic action, space or, more precisely, time-space, can become communication, signification, or both, at the extreme. In this time-space, the interaction between signs, constituted in a material bearer (representamen) is produced, and from the content of information (uniting the sign's other two terms, object and interpretant), is produced the content expressed here in terms peculiar to statistical-not semiotic-analysis (in particular, in units of information and not in those qualities contained in the meaning produced by signs). We refer to Peirce's concept of sign, but we can extend the model to any system defined triadically, especially any sign seen as relation.

Shannon's model implies the informational field but does not define it, although the premises for this definition can be found in this model. The criticism made by Meyer-Eppler (1969), Watzlavik, Beavin, and Jackson (1967), and others considers only the fact that the distinction between information (and informing) and communication is necessary from the methodological viewpoint. Unfortunalety, the solutions proposed in such critical analyses sometimes repeat Shannon's error. One example of this is Meyer-Eppler's three types of communication chains: linguistic, observational, and diagnostic; another is Watzlavik's connection between message and communication.

We remain on the informational, syntactic level. The interaction of signs, expressed in the stochastic process, is simplified. Signs are considered independent of their meaning. Here we must insert a historic note. Weaver (whose text was first published in 1949 in "Scientific American") tends to complete Shannons's initial model, discussing the three levels of communications problems: technical, semantic, effective. Weaver notes the association of information to a source (the consequence being the statistical study of language structure) and 
infers: "The idea of utilizing the powerful body of theory concerning Markoff processes seems particularly promising for semantic studies, since this theory is specifically adapted to handle one of the most significant but difficult aspects of meaning, namely the influence of context." (Shannon, 1959, p. 117). We consider this conclusion surprising not because it does not correctly characterize the Markov processes but because the one-directional model Weaver refers to actually has no semantic dimension. Signs, in the sense we attribute to them, do not exist at the informative level, but signals, characterized in their succession through order relations, which have meaning only on the level of unmediated material reality, a reality that the sign transcends (which even constitutes its reason for being). The semantic dimension takes shape just when this transcending takes place, when information becomes the content of communication and the sign holds the place of the concrete, the immediate, the direct, that is, when the transition from signal to sign and from the one-directional informational flow to a bi-directional connective system takes place. These are necessary but not sufficient conditions. The bi-directionality of a telemechanical system, for example (which we give because after de Mauro (1966) and Eco (1976), it turns into stereotype despite several shortcomings), does not give evidence of the meaning of the sequence of signal commands. The confirmation of signal sequence, of code (the correct reception from an technical viewpoint and the execution of given commands) does not transform the above mentioned system from an informational into a communicational one. This is a matter of a univocally determined system of signals, and not of a semiotic system that implies the interpretant subsign. There is no transition from signal to sign since the interpretant subsign per se does not exist but is simulated by the command-execution mechanism. The code impressed has a fixed structure to which a specific function is associated. In a technical sense, any equivocation is eliminated. In order to achieve this desideratum, the system is submitted to a continuous "closing," that is, made less and less sensitive to context (progressive codification is the direct effect of this desensitizing to context), to environment (including transmission channels).

The sign exists only in and through a context of signs of the same nature, of a different nature, or in and through the unhomogenous context of reality in its complexity. The sign is not necessarily the bearer of information, but it is necessarily a means of each process of communication and signification.

Definition 1.2: The semiotic field is the unity between the fields of communication and signification.

Definition 1.2.1: The semiotic field of communication is the field attached to an indeterminate bi-directional informational system.

Definition 1.2.2: The semiotic field of signification is the para-communicative field attached to an indeterminate bi-directional informational system.

These definitions must be justified and verified in practice. Referring to the definition of sign as intermediary, evidently a triadic definition, one which the mathematical model of the category reflects in its essence, we must understand 
why, in order to speak of communication, we must consider bi-directional indeterminate systems that can model the interaction of the signs of the semiotic system. The indetermination emphasized in 1.2.1 and 1.2.2 means that there is no question of two sign-independent flows that circulate in opposite directions but of systems in which signs influence one another in the constitution of consistent semantic units in each direction in part as well as in the two directions. This is a matter of a complex semiotic connection which is not reduced only to reactions (question/answer, for instance) but comprises reciprocal, continuous, optimizing actions. Signs are continuously controlled in relation to functioning, in view of realizing meaning, both on the communicative and significative levels. This condition was not kept in mind in Shannon's model. The criticism the latter has received tends only towards perfecting its presentation but not towards completing it in the above sense.

To deal with communication as a semiotic process, we must first concern ourselves with the structure of the process. Proceeding in the spirit of Peirce's semiotic-in which man himself is a sign-we understand that the Emitter-Receptor distinction cannot be accepted even as a functional representation. Reception is not the result of reversing the emission function. In any act of emission, reception is present; in any act of reception, emission is influenced. At the ends of the communicational system the two functions are reunited, when people, not technical installations, are involved.

Here a primary element too important to be ingnored intervenes: man's perceptive capacity is gigantic (in informational units, approximately $10^{35}$ to $10^{41)}$ bits per second): his emitive capacity (conscious output) is very small (50 bits per second, cf. Marko, 1967). It is true that perception involves global information (sometimes accidental, entropic, unstructured, etc.) while emission is "directioned," structured, essential information. Signs optimize the man-nature (manenvironment) relationship. When the semiotic system of culture is established, it acts protectively, as a filter, helping man to retain not so much quantities as qualities. Signification appears only at this level of evolution. Each human "generator" holds a finite quantity of information (innate and acquired). The accumulation and transmission of this information is represented through a stochastic process characterized through its entropy at the extreme. Connection follows a law of sychronization, also stochastic. We can no longer speak of information (passively accepted, one-directional) but of transinformation. In this case, entropy characterizes independent flows on the one hand, and on the other, interdependent flows that actually define communicative content. 
Figure 2

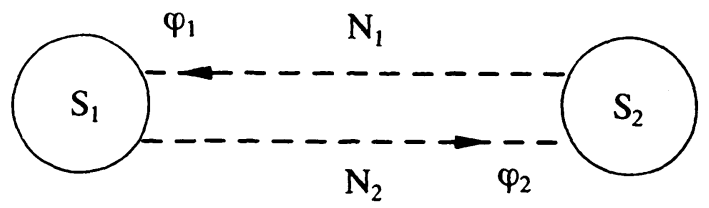

$$
\left\{\begin{array}{l}
\varphi_{1}=\mathrm{f}\left(\varphi_{2}\right) \\
\varphi_{2}=\mathrm{g}\left(\varphi_{1}\right)
\end{array}\right.
$$

defined as interdependence.

Sign-flows $\varphi_{1}$ and $\varphi_{2}$ are related. The two channels of communication (media in the broad sense of the word) can be indentical or different. The possible action of sources of different "noise" associated to each channel in part (sometimes to each emitter in part) is expressed in their separate configuration. A simple example is communication through correspondence. The possible sources of "noise" (which do not necessarily involve both persons in the communicative semiotic field) would be the level of mastery of written language, the quality of the material used, the level of postal technology, open or hidden censorship, etc. It should be pointed out that it is not always easy to distinguish between information and noise.

Let us now turn not to the technical aspect that Shannon had in mind but to the semiotic aspect and thus to the problem in principle of codes. In the given example, a lack of skill in written language (the "noise" of orthographic errors, for example) can impose itself over the effect of codification in relation to known censorship. Likewise, the "noise" of censorship can dictate an intentional modification of information. In the case of the use of two different sign systems (languages, in particular), other implications arise (upon which we will not dwell here). It is important to point out that the human subject receives information on afferent channels (where decodification also takes place as the primary form of reducing the informational flow) and transmits information on an efferent channel, foreseen as an output system containing a codifier. To be as rigorous as possible, we should point out that neurophysiological research has demonstrated the connection between the two channels, the influence between codification and decodification, and the direct participation of reception in emission and vice versa, aspects which the model under discussion will not retain. There are other such aspects regarding the auto-active nature of the human emitter (memorization as a generative process, spontaneous neural activity such as $\alpha$-rhythms, learning, forgetting, etc.) which, though important from a semiotic perspective, can be very difficult to express at the current stage of mathematical or other formalization. In time, they too will be included in our model. 
The emitter and receptor reach a contradictory unit $\sum$. The human processes of communication, as complex semiotic processes, have shown that this unit, within the reality of these processes, has great variety (which is sometimes defined as subjectivity). The modeling we pursue concerns the structure of the process, not individual aspects. This structure (Figure 3) can be processualy detailed. In a first development, we can reproduce Shannon's initial model in double:

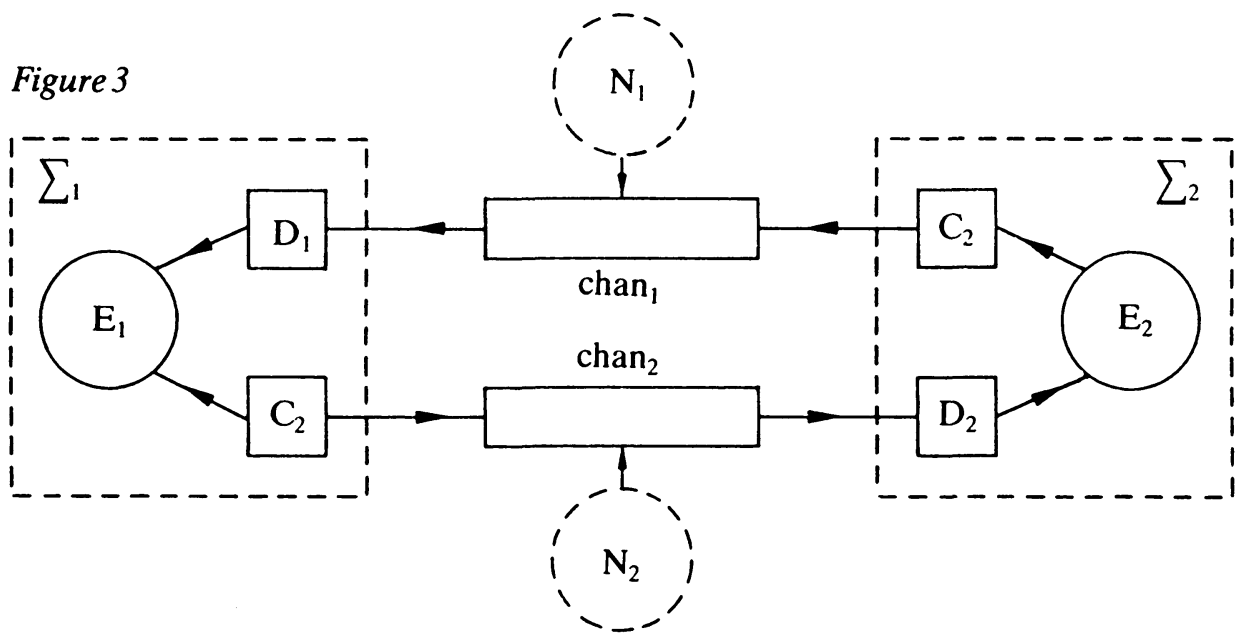

We indicated the differentiation $E_{1}, E_{2}, C_{1}, C_{2}, D_{1}, D_{2}$, $\operatorname{chan}_{1}$, chan ${ }_{2}, N_{1}, N_{2}$, but we can renounce them on the basis of the structural model of information processing (Figure 2). In certain cases, the differentiation of channels can also be renounced (when the same medium is used, in direct, telephonic, or telegraphic communication, for example) but not the differentiation of noise sources. These, we repeat, are actually only schematically represented, independent of the emitter as such. In reality, an important link exists between them, expressed through the emitter's semiotic sensitivity to context (environment).

In some analyses, a transition from sign to signal and then back to sign can be observed in processes of information transmission:

Figure 4

noise

source $\rightarrow$ transmitter $\rightarrow$ signal $\rightarrow$ channel $\rightarrow$ signal $\rightarrow$ receptor $\rightarrow$ message $\rightarrow$ destination 
This transition (for example, a modeling in amplitude of frequency as in radio or television), although important as a premise of communication, does not concern communication but the technical conditions of its production. The signal appearing in the scheme is the bearer of a complexly structured set of signs, the process described having a double codification: technical (transmissional) and semiotic (interpretational). The mathematical characterization (which we do not reproduce here) concerns the informational aspects of communication, information itself being probabilistically defined. The absence of educational elements (the results of considering stationery processes, in particular learning and forgetting) is not the only limitation such a mathematical representation accepts.

Definition 2: The semiotic field of noise is called constraint.

Definition 2.1: The degree of sensitivity to the noise field is called independence.

Definition 2.1.1: The contradictory unit between constraint and independence (degree of insensitivity to noise) defines the semiotic system's degree of liberty.

Definition 3: The semiotic field of context is called second-degree constraint.

Definition 3.1: The degree of sensitivity to the contextual semiotic field is called the contextual independence of the semiotic field.

Definition 3.2: The contradictory unity between second-degree constraint and contextual independence (degree of insensitivity to context) defines the semiotic system's degree of contextual liberty.

Definition 4: Dependence on noise and context determines the degree of the semiotic system's openness.

In connection to these definitions, it should be pointed out that in the real dynamics of semiotic processes, the signs that participate in semioses are determined both in the system and outside it. In its ideality, the semiotic process is a process of signs; in its reality, what is presented to us as a result of sign processes (repertory, syntactic rules) is actually the expression of constraint by the semiotic system, more precisely, by the semiotic field of noise, of context. Meaning itself, as the final expression of the sign's realization, reflects this situation. In a first instance, we could consider the semiotic field of noise as a component of the context per se and the semiotic context outside the sign system as such immediately appears. On the one hand we have an internal connection which can be described in the terms of the generative theory; on the other, the external connection that is only indirectly and very complexly reflected in the result of the semiotic process.

Let us take the case of an ensemble (text, painting, song, or any other). The fact that there is a necessary connection between the words of a literary discourse, or between the components of a painting (form and colour are usually mentioned), or between the sounds and words making up a song (not the only connection possible but basically one of the optimal from the perspective of aesthetic effect) is unanimously accepted. This is the internal connection specific to the semiotic system. But in its realization as a form of communication (between 
author and future public) and signification, the ensemble is conditioned not only by the signs and rules of association it contains but especially by those which, though not contained, not directly obvious, impose a certain choice and not another: the choice made and not one from among the multitude of those possible. Society, for instance, does not act as an abstract, an outer force on the artist. Its action is complex, but in direct connection to the product of the artist's activity. It is a semiotic action just as the action of other factors (economic included). Referring now to the model of communication (Figure 4), we observe that it corresponds to a closed system in whose framework $\sum_{1}$ and $\sum_{2}$ represent two subsystems that enter into connection.

\section{Figure 5}

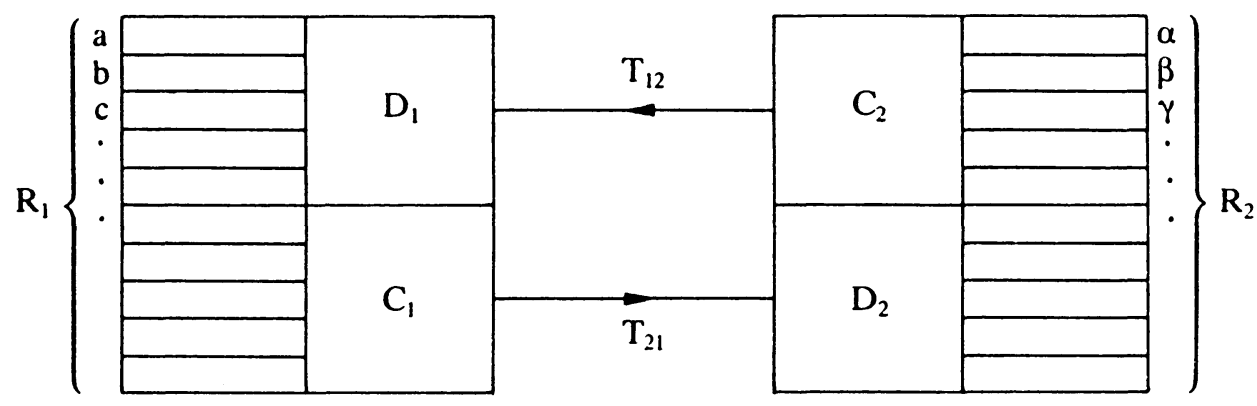

The determination of sign succession-considering only the two distinct repertories $R_{1}$ and $R_{2}$ (a simplification that does not affect the image proposed)-ignores the action of the environment, of the semiotic field of noise, that is. It would be necessary to determine the differential probability of the appearance of the signs of other processes which, though going on in other systems (social, political, economic, etc.), represent a context (necessary or accidental) of semiotic processes, a factor influencing the field. The entropy of the semiotic system is practically determined in this way. On the one hand we have a neg-entropic action that institutes a meaning by structuring a coherent set; on the other is a contrary, intensely entropic action that destabilizes the system, deforms the field, negates its structure, and imposes transition to another structure, another state of the field. Codification within the system's framework is the expression of resistance to the effect of noise. Any code has the effect of stabilizing; any act of codification is neg-entropic and represents a modality of closing the system, hence of imposing the principle of contemporaneity in the semiotic field. The signs of the semiotic field of noise act on the unity between message and code, which can be perfect only when the message is identical to the code (for example, magic formulas or abstract mathematical expressions). Actually, it is a matter of interaction. A tendency towards stabilization is noticed on the one hand, the negation of 
stability, structure, and the associated function on the other. If civilization truly represents the stabilized part of a culture, then it is somehow clear that it represents a quasi-closed semiotic system. In contrast, culture is an open semiotic system that evolves in the environment of civilization as a socio-historic given as well as in the environment of the dynamic field of forces it negates. Therefore, the assertion that culture is the product of noise (in the sense given above) is not only metaphoric (as Fabri, (1973) believes).

Learning (and forgetting, both elements that our model does not represent), while going on in relatively closed systems (the institutionalized nature of the forms of education), is also determined by noise, more precisely, by its semiotic field. We thus return to the problem posed by Shannon's research, the problem of the influence of the relation between information and noise (their optimal relation) to a received signal:

$$
E=f(S, N)
$$

in Shannon's notation, $\mathrm{E}$ is the received signal, $\mathrm{S}$ is the transmitted signal, and $\mathrm{N}$ is noise (1959, p. 34). In a first analysis, Shannon considers that noise has the same nature (probabilistic) as the (transmitted) message, hence representable through a stochastic process. Later, he goes from the discrete to the continuous model. In the case of semiotic processes, we deal with complex processes in which discrete elements and elements of continuity are perpetually combined. The question is not one of optimal dimensioning of the channels of communication but of real interaction between the two semiotic fields of communication and signification. We thus arrive at the need to define them, bearing in mind the way they are constituted and the way their interaction really takes place. In this sense, it is useful to describe field (to utilize the premise of the categorical analysis of the sign) and to define semiotic processes as field processes.

The triadic structure we have in mind as an implicit given of the field (because it is a datum of the sign) is already characteristic for its categorical definition. The sign stands for something that exists (which Peirce called object) and brings about a relation. What exists is an elementary notion. Mario Bunge believes that only two entities are elementary by nature (he uses the word "simplicity"): things and signs. The sign can be defined as a unit between relation (which de Saussure's sign reflects) and function (in Mukarovsky's an Lotman's sense). As relation, the sign has a trichotomic (or superior, reducible to triadic, however) structure. As functional representation, it represents the existent in a triadic sign relation. This double nature of the sign-which Peirce's semiotic does not exhaust but only embodies in one of its possible forms (with the respective sign typology attached to it)-especially interests us when we speak of the semiotic field. As relation between two irreducible entities, the sign is only the triadic unit and realizes a functional representation of the unit between what exists and how this existence is perceived through signs. Semiotics consequently represents the system (structures and states that can be determined) of functional relations through which man's generic representations are realized. It is therefore a matter of an intertheoretical 
domain with heuristic and maieutic dimensions (as defined by Socrates) prescriptive and pragmatic, axiomatic. Field Theory can be applied to the whole domain as well as to the study of communication and signification.

\section{Bibliography}

Bridgman, P. W., The Nature of Some of Our Physical Concepts, New York 1952. Eco, Umberto, La struttura assente, Milano 1968.

-, Einführung in die Semiotik, München 1972.

-, A Theory of Semiotics, Bloomington 1976.

Fabri, Paolo, Le communicazioni di massa in Italia: sguardo semiotico e malocchio della sociologia, in: Versus 5, 1973.

Hartman, RobertS., Can Field Theory be Applied to Ethics?, Northwestern University, Dissertation (unpublished), 1946.

Lewin, Kurt, Field Theory and Experiment in Social Psychology Concepts and Methods, in: American Journal of Sociology, 44, 1939, pp. 868-896.

-, Field Theory in Social Science (Selected Theoretical Papers), New York 1952.

Lotman, I. M., Lektsii po structural'noi poetiki: Vvedenie, Teoria Stikha, (Lectures on Structural Poetics: Introduction, Theory of Verse), Brown Universitiy Slavic Reprint V, Providence 1968.

Marko, Hans, Information Theory and Cybernetics, in: IEE Spectrum, 1967, p. 79. Mauro, Tulio de, Modelli semiologici, l'arbitrarità semantica, in: Lingua e stilo, 1, 1966. Meyer-Eppler, W., Grundlagen und Anwendungen der Informationstheorie, Berlin 1969. Shannon, Claude E., The Mathematical Theory of Communication, in: Mathematical Theory of Communication. Urbana 1959, pp. 3-94.

Spivak, Michael, Differential Geometry, Boston 1970.

Watzlavik, P., J. H. Beavin, D. Jackson, Pragmatics of Human Communication. A Study of

Interactional Patterns, Pathologies, and Paradoxes, New York 1967.

Weaver, Warren. Present Contributions to the Mathematical Theory of Communication, in:

The Mathematical Theory of Communication, Urbana 1959, pp. 94-117.

\section{Summary - Résumé - Zusammenfassung}

The complexity of communication processes presupposes the continual improvement of the methods and perspectives dealing with them. This is not a matter of terminological adaptation or technical innovation, but of understanding the complex mechanism through which communication takes place in social and other communication-sensitive contexts. Continuing Robert Hartman's line of thought (expressed in his still unpublished dissertation "Can Field Theory be Applied to Ethics?" Northwestern University, 1946), the article sets communication in a dynamic framework; that is, it relates sign processes to the conceptual and practical aspects of interactions as reflected in the gnoseological model of field. The results of the scientific field theory are applied to communication phenomena perceived in cultural context without reducing them to physical reality but suggesting analogy and modelling procedures useful in the context of computer-aided communication. Analogies are possible between the way people interact in the semiotic field with the various signs they come upon and the complementarity of field and quanta. Effective formal descriptions, such as those provided in the quantum theory, of these interactions 
might prove useful in better understanding communication and in designing improved communication networks.

La complexité des processus de la communication implique la nécessité permanente d'améliorer les méthodes et les perspectives par lesquelles nous les approchons. Il ne s'agit pas d'une simple adaptation de terminologie ni d'une innovation the technique; il s'agit de comprendre les méchanismes complexes par lesquels la communication s'établit dans des contextes sociaux aussi bien que dans autres contextes qui influencent la communication. Suivant la pensée de Robert Hartman (exprimée dans sa dissertation toujours non-publiée («Can field theory be applied to ehtics?», Northwestern University, 1964), cet article met la communication dans un cadre dynamique; c'est-à-dire, il met en relation les processus du signe et les aspects conceptuels et pratiques des interactions reflétées dans le modèle gnoséologique du champ. Les résultats de la théorie scientifique de "champ» s'appliquent aux phenomènes de la communication perçus dans le contexte culturel sans les réduire à la réalité physique, mais suggérant une analogie et modéllant des processus que servent dans le contexte de la communication aidée par ordinateur. On peut faire des analogies entre la manière de comportement des gens dans le champ sémiotique avec les signes qu'ils rencontrent et la complémentarité du champ et quanta. Descriptions formelles des interactions (comme celles offertes par la théorie de quanta) peuvent se montrer efficaces pour mieux comprendre la communication et pour développer de meilleurs réseaux de communication.

Die ständig zunehmende Komplexität der Kommunikationsprozesse erfordert nicht nur die kontinuierliche Beschäftigung mit den Methoden und Perspektiven der Thematik, sondern deren laufende Verbesserung. Dies ist weniger eine Frage von terminologischer Anpassung oder technologischer Innovation, sondern vielmehr des Verständnisses der komplizierten Mechanismen, die Kommunikation in den verschiedensten sozialen und sonstigen kommunikations-empfindlichen Situationen ermöglichen. Der Beitrag geht von Robert Hartmans Versuch (ausgedrückt in seiner bisher nicht veröffentlichten Dissertation „Can Field Theory be Applied to Ethics?“", Northwestern University, 1946) aus, doch stellt er die Problematik in einen dynamischen Rahmen, der Zeichenprozesse in Abhängigkeit von konzeptionellen und praktischen Aspekten sieht, ähnlich wie beim gnoseologischen Feld-Modell. Die Erkenntnisse der wissenschaftlichen Feld-Theorie werden auf kulturabhängige Phänomene der Kommunikation projiziert - weniger in der Absicht physikalischer Materialisierung, sondern mit dem Ziel, Analogien und Modelle aufzuzeigen, die sich für computer-unterstützte Kommunikation eignen. Analogien sind denkbar zwischen der gegenseitigen Beeinflussung von Mensch und Zeichen, bzw. dem Prozeß der Wahrnehmung im semiotischen Feld und der Theorie der (wechselseitigen) Beziehung von Feld und Quanten. Wirksame Beschreibungen solch gegenseitiger Beziehungen, wie beispielsweise aus der Quanten-Theorie bekannt, könnten sich als nützlich für ein besseres Verständnis der Kommunikation und für die Entwicklung besserer Kommunikations-Systeme erweisen. 
